Notre Dame Journal of Formal Logic

Volume 35, Number 4, Fall 1994

\title{
Topological Structure of Diagonalizable Algebras and Corresponding Logical Properties of Theories
}

\author{
GIOVANNA D'AGOSTINO
}

\begin{abstract}
This paper studies the topological duality between diagonalizable algebras and bi-topological spaces. In particular, the correspondence between algebraic properties of a diagonalizable algebra and topological properties of its dual space is investigated. Since the main example of a diagonalizable algebra is the Lindenbaum algebra of an r.e. theory extending Peano Arithmetic, endowed with an operator defined by means of the provability predicate of the theory, this duality gives the possibility to study arithmetical properties of theories from a topological point of view. We find topological characterization of $\Sigma_{1}$-sound theories and of sentences that are $\Sigma_{1}$-conservative over such a theory.
\end{abstract}

1 Introduction The equational class of diagonalizable algebras was introduced in the 1970s in order to study properties of arithmetical theories from an algebraic point of view.

Definition 1.1 A diagonalizable algebra is a pair $(D, \square)$, where $D$ is a Boolean algebra with operations $\perp, \top, \wedge, \neg, \vee$, and $\square$ is a map from $D$ to $D$ satisfying the following identities:

1. $\square \top=\top$;

2. $\square(a \wedge b)=\square a \wedge \square b$;

3. $\square(\square a \rightarrow a)=\square a$;

where, as usual, $a \rightarrow b$ is $\neg a \vee b$. The operator $\diamond$ defined as $\diamond a=\neg \square \neg a$ is also used, and in this case a diagonalizable algebra is considered to be a pair $(D, \diamond)$.

The main example of a diagonalizable algebra is the Lindenbaum algebra $D_{S}$ of an r.e. theory $S$ extending Peano Arithmetic $P A$, with the $\square$ defined as $\square[p]=$ Received August 6, 1992; revised August 16, 1994 
$\left[\right.$ Theor $\left._{S}(\bar{p})\right]$. Here $[p]$ denotes the equivalence class of the sentence $p$ with respect to provable equivalence in $S$, while Theor $_{S}(x)$ is the provability predicate of $S$.

Many results prove the class of diagonalizable algebras to be adequate for the study of arithmetical theories: among them, we recall that, according to Solovay's Theorem, the diagonalizable algebra of $P A$ generates the variety of diagonalizable algebras. Besides, a recent result of Shavrukov (see Shavrukov [10]) shows how the algebraic structure is sensitive to the change of the base theory, e.g., the diagonalizable algebras of $P A$ and $Z F$ are not isomorphic (while they can be recursively embedded each one into the other).

During the 1980s, the topological duality between Boolean algebras and Stone spaces was extended to diagonalizable algebras. First, Esakia proved that if a topological space $X$ is scattered (i.e., every nonempty subset of $X$ contains an isolated point), we get a diagonalizable algebra by defining the operator $\diamond$ on the Boolean algebra $P(X)$ as the derived set operator $d$ of the topology; moreover the class of these "topological" algebras generates the whole equational class of diagonalizable algebras. Later, Abashidze showed that the ordinal $\omega^{\omega}$ with the interval topology is generic in the class of diagonalizable algebras (see Abashidze and Esakia [1] and also Blass [3]).

A duality is obtained as follows. Given a diagonalizable algebra $(D, \diamond)$, consider the Stone space $\left(D^{*}, T\right)$ of the underlying Boolean algebra (as usual, the clopen sets of the Stone space are identified with the elements of $D$ ). On this space define a relation $R$ as follows: $x R y$ iff the ultrafilter $x$ contains the set $\{\diamond a / a \in y\}$. We call $R$ the dual relation of $(D, \diamond)$ and for each clopen set $a$ of the Stone space $\left(D^{*}, T\right)$, the clopen set $\diamond a$ equals $R^{-1}(a)$ (see Magari 7 ).

Definition 1.2 From now on, the word "clopen" always refers to a clopen set in the Stone topology.

Let us consider in particular the Stone space of $D_{P A}$. Here, ultrafilters can be identified with complete theories extending $P A$, and the relation $R$ has a logical meaning: $x R y$ iff the theory $x$ proves the consistency of all the theories $P A+a$ for each sentence $a$ belonging to the theory $y$. Note that an ultrafilter $x$ is reflexive (with respect to the relation $R$ ), iff the complete theory $x$ proves the reflection principle Theor $_{P A}(a) \rightarrow a$ for each sentence $a$.

To obtain a topological duality for diagonalizable algebras, once $(D, \diamond)$ is given, define a new topology $\bar{T}$ in the Stone space $D^{*}$ by considering as open sets the $R$ hereditary sets (i.e., the subsets $H$ of $D^{*}$ with the property that $x \in H$ and $x R y$ imply $y \in H$ ); in this topology, the interior of a clopen $p$ is given by the set $p \wedge \square p$. The topology $\bar{T}$ turns out to be relatively scattered with respect to the Stone topology, that is, each clopen set in $T$ contains an isolated point in the topology $\bar{T}$. It can be proved that the "topological" diagonalizable algebra, obtained by considering the clopen sets with the derived set operator $d$ of the $\bar{T}$-topology as $\diamond$, is isomorphic to the diagonalizable algebra $(D, \diamond)$ (see Bernardi and D'Aquino [2]).

In this paper, the correspondence between algebraic properties of diagonalizable algebras and topological properties of the dual space is investigated. By restricting this study to the case of diagonalizable algebras of theories, we find topological characterization of $\Sigma_{1}$-sound theories and of sentences that are $\Sigma_{1}$-conservative over such 
a theory (see Corollary 3.5 and Theorem 4.3).

In order to prove these results in full generality we recall the definition of net; we note that this definition would be unessential if we were dealing only with diagonalizable algebras of theories, because in this case the topology $\bar{T}$ of the dual space would be second countable and the definition of sequence would be enough to describe the properties of the topology.

Definition 1.3 A directed system is a set $S$ together with a transitive relation $\prec_{S}$, such that, if $\alpha, \beta \in S$, there is a $\gamma \in S$ with $\alpha \prec_{S} \gamma$ and $\beta \prec_{S} \gamma$.

Definition 1.4 A net is a map from a directed system $S$ into a topological space $X$. We write $x_{\alpha}$ for the value of the net at $\alpha$ and $\left(x_{\alpha}\right)_{\alpha \in S}$ for the net itself.

Definition 1.5 A net $\left(x_{\alpha}\right)_{\alpha \in S}$ is said to converge at $x \in X$ if for each open set $O$ containing $x$ there is $\alpha \in S$ such that $x_{\beta} \in O$ if $\alpha \prec_{S} \beta$. In this case we call $x$ a limit point of the net $\left(x_{\alpha}\right)_{\alpha \in S}$. If the topologial space is not Hausdorff, it is possible for a net to converge to more than one point.

Definition 1.6 A net $\left(y_{\beta}\right)_{\beta \in T}$ is a subnet of a net $\left(x_{\alpha}\right)_{\alpha \in S}$ if:

1. there is a map $\varphi$ on the directed system $T$ with values in $S$ such that $y_{\beta}=x_{\varphi \beta}$, for each $\beta \in T$;

2. for each $\alpha \in S$ there is a $\beta \in T$ with the property that, if $\beta \prec_{T} \beta^{\prime}$, then $\alpha \prec_{S} \varphi \beta^{\prime}$.

We may describe a topological space $X$ by using nets; in fact, a subset $C$ of $X$ is closed iff it contains all limit points of nets with values in $C$, while a point $x$ is a cluster point for a subset $Y$ iff there is a net in $Y-\{x\}$ that converges to $x$. We also recall that compact spaces are exactly those topological spaces in which every net has a convergent subnet.

Let $D$ be a diagonalizable algebra. The set $D^{*}$ of all ultrafilters of $D$, endowed with the Stone topology and with the topology $\bar{T}$ described above, is called the dual space of the diagonalizable algebra $D$. If $R$ is the dual relation on $D^{*}$, then the following proposition is proved in D' Agostino 44:

Proposition 1.7 Let $x, y$ be elements of $D^{*}$. Then

$$
x \text { Ry iff there is a net }\left(x_{\alpha}\right)_{\alpha \in S} \text { such that } \begin{cases}\forall \alpha \in S x_{\alpha} \neq x \\ \left(x_{\alpha}\right) \rightarrow x \text { in the topology } \bar{T} \\ \left(x_{\alpha}\right) \rightarrow y \text { in the topology }\end{cases}
$$

In particular, an element $x$ of $D^{*}$ is reflexive iff there is a net of elements different from $x$ that converges to $x$ in both $T$ and $\bar{T}$.

We recall that a diagonalizable algebra $D$ is said to be $\omega$-consistent (see Magari [6]) if $\top \neq \perp$, and $\square a=\top$ implies $a=\top$.

From the dual point of view, it is easy to see that an algebra is $\omega$-consistent iff $R^{-1}(x) \neq \varnothing$ for each point $x$ in $D^{*}$ (for a logical counterpart of $\omega$-consistency, see Proposition 2.2.

In D'Agostino [4] the following proposition is proved:

Proposition 1.8 $D$ is $\omega$-consistent iff $D^{*}$ is the only $\bar{T}$-open set containing the set of reflexive points. 
2 First results A diagonalizable algebra $D$ is said to possess the disjunction property (see [9]) if $\square a \vee \square b=\top$ implies $\square a=\top$ or $\square b=\top$ (equivalently, $\diamond p \wedge \diamond q$ is different from $\perp$ if $\diamond p, \diamond q$ are different from $\perp$ ).

$D$ is said to have the strong disjunction property (see [9]) if it is $\omega$-consistent and has the disjunction property, that is, $\square a \vee \square b=\top$ implies $a=\top$ or $b=\top$ (equivalently, $\diamond a \wedge \diamond b$ is different from $\perp$ if both $a$ and $b$ are different from $\perp$ ). In the class of diagonalizable algebras the $\omega$-consistency and the disjunction property are independent from each other, as one can see by considering the following:

Example 2.1 The direct product $F \times F$ of a free algebra $F$ (for instance on the empty set) with itself is $\omega$-consistent but does not have the disjunction property, while any finite algebra with the disjunction property (e.g., any finite diagonalizable algebra where $\square a=\top$ for all $a$ ) is not $\omega$-consistent.

The situation is different if we consider diagonalizable algebras of theories, where the following holds:

Proposition 2.2 (See Shavrukov [9) $\quad S$ is $\Sigma_{1}$-sound iff the diagonalizable algebra $D_{S}$ is $\omega$-consistent iff $D_{S}$ has the strong disjunction property.

We first intend to characterize the disjunction property and the $\omega$-consistency from a topological point of view for all kinds of diagonalizable algebras; we will see later that these characterizations become simpler when considering diagonalizable algebras of theories.

Theorem 2.3 $D$ is $\omega$-consistent iff the set of points which have a nonempty $\bar{T}-$ derived set is dense in the Stone space.

Proof: Let $d$ be the derived set operator of the topology $\bar{T}$. Notice that $R^{-1}$ $(y)-\{y\}=d\{y\}$. If $D$ is $\omega$-consistent and $d\{y\}$ is empty, then $R^{-1}(y)$ is equal to $\{y\}$, and Proposition 1.7 guarantees the existence of a net $\left(y_{\alpha}\right)$, with $y_{\alpha} \neq y$ for every $\alpha$, that converges to $y$ in both topologies; since eventually the set $d\left\{y_{\alpha}\right\}$ is not empty, we conclude that $y$ belongs to the closure (in the Stone topology) of the set $\{x / d\{x\} \neq \varnothing\}$.

Vice versa, if there is a net $\left(y_{\alpha}\right)$ in $\{x / d\{x\} \neq \varnothing\}$ that converges in the Stone topology to $y$, then for every $p_{1}, \ldots, p_{n}$ with $p_{i} \in y$, there is an $\alpha$ such that $p_{1}, \ldots, p_{n}$ are in $y_{\alpha}$. But $R^{-1}\left(y_{\alpha}\right)$ is not empty, thus we have $\diamond p_{1} \wedge \ldots \wedge \diamond p_{n} \neq \perp$, and the set $\{\diamond p / p \in y\}$ has the finite meet property as set of elements of the Boolean algebra $D$; thus, there exists an ultrafilter containing the set $\{\diamond p / p \in y\}$ and this implies $R^{-1}(y) \neq \varnothing$. Since this is true for all points in the dual space, the algebra is $\omega$-consistent.

Lemma 2.4 The following conditions are equivalent:

(i) D has the disjunction property;

(ii) there is a point $z$ in the dual space such that $z R x$ for each $x$ with $R^{-1}(x) \neq \varnothing$;

(iii) if $R^{-1}(x) \neq \varnothing$ and $R^{-1}(y) \neq \varnothing$, then the set $R^{-1}(x) \cap R^{-1}(y)$ is not empty.

In particular, $D$ has the strong disjunction property iff $R^{-1}(x) \cap R^{-1}(y)$ is not empty for every $x, y$ in $D^{*}$, iff there exists a point $z \in D^{*}$ such that $z R x$ for all $x \in D^{*}$. 
Proof: (i) $\rightarrow$ (ii) Using induction we can prove that the disjunction property implies the $n$-disjunction property, that is, if $\diamond p_{i} \neq \perp$, for $i=1, \ldots, n$ then $\diamond p_{1} \wedge \ldots \wedge$ $\diamond p_{n} \neq \perp$ (see Friedman [5]). This implies that the set $\{\diamond p / \diamond p \neq \perp\}$ has the finite meet property, thus it is contained in an ultrafilter $z$; but if $z \supseteq\{\diamond p / \diamond p \neq \perp\}$, then $z$ satisfies (ii).

(ii) $\rightarrow$ (iii) Obvious.

(iii) $\rightarrow$ (i) If $\diamond a, \diamond b$ are both different from $\perp$, two ultrafilters $x, y$ exist such that $a \in x, b \in y$ and $R^{-1}(x) \neq \varnothing, R^{-1}(y) \neq \varnothing$; from (iii) it follows that $R^{-1}(x) \cap R^{-1}$ $(y) \neq \varnothing$, and $\diamond a \wedge \diamond b \neq \perp$.

Remark 2.5 From the Lemma we see that the disjunction property is equivalent to the existence of an ultrafilter $y$ such that if $\square a \in y$, then $\square a=\top$; then the strong disjunction property is equivalent to the existence of a $y$ such that $\square a \in y$ implies $a=\mathrm{T}$, that is, $y$ "behaves like" the ultrafilter of the true sentences (in the standard model) of $P A$.

From a topologial point of view, since $y R x$ for all $x \in D^{*}$, the point $y$ belongs to the closure of every point of the space, or equivalently, the only open set containing $y$ is the whole space. We refer to this $y$ as an "antigeneric point."

Theorem 2.6 $D$ has the disjunction property iff every net in the set $\left\{x / R^{-1}(x) \neq\right.$ $\varnothing\}$ converges.

Proof: $(\rightarrow)$ It follows easily from Lemma 2.4part (ii).

$(\leftarrow)$ Given $x, y$ such that $R^{-1}(x) \neq \varnothing, R^{-1}(y) \neq \varnothing$, let $\left(x_{n}\right)$ be the sequence that takes alternatively the values $x, y$, and let $z$ be its limit; thus $\{x, y\} \subseteq\{z\} \cup R(z)$, and only one of the following cases occurs:

(i) $x=y$;

(ii) $x \neq y, x=z$ and $y \in R(z)$ (or the symmetrical case);

(iii) $x \neq y,\{x, y\} \subseteq R(z)$.

A simple verification shows that in every case the set $R^{-1}(x) \bigcap R^{-1}(y)$ is not empty, and thus $D$ has the disjunction property according to Lemma 2.4 part (iii).

Remark 2.7 Using Lemma 2.4 we can prove something more: $D$ has the disjunction property iff there is a point in the dual space to which every net of the set $\left\{x / R^{-1}\right.$ $(x) \neq \varnothing\}$ converges.

It follows that if $D$ has the strong disjunction property then every net in $D^{*}$ converges (to the antigeneric point of Remark 2.5; see Theorem 3.1 for the converse).

Theorem 2.8 $D$ has the strong disjunction property iff every nonempty closed set in the space $\left(D^{*}, \bar{T}\right)$ is connected and contains a reflexive point.

Proof: $\quad(\rightarrow)$ If $C, C_{1}, C_{2}$ are nonempty closed sets in $\left(D^{*}, \bar{T}\right)$ such that $C=C_{1} \cup$ $C_{2}$, let $x \in C_{1}, y \in C_{2}$. We have $C_{1} \cap C_{2} \supseteq R^{-1}(x) \cap R^{-1}(y)$, and this last set is not empty according to Lemma 2.4. This implies that each closed set $C$ in $\left(D^{*}, \bar{T}\right)$ is connected; since $D$ is $\omega$-consistent, each nonempty $\bar{T}$-closed set contains a reflexive point (see Proposition 1.8 .

$(\leftarrow)$ If $a, b$ are different from $\perp$, let $x, y$ be two ultrafilters containing respectively $a$ and $b$. In the dual space $\left(D^{*}, \bar{T}\right)$, the sets $C_{1}=\{x\} \cup R^{-1}(x)$ and $C_{2}=$ 
$\{y\} \cup R^{-1}(y)$ are closed and not empty, and according to the hypotheses there is a reflexive point $z$ in $C_{1} \cap C_{2}$. Since $z$ is reflexive, we have $z \in R^{-1}(x) \cap R^{-1}(y)$, and since $\diamond a \wedge \diamond b \in z$, we conclude that $\diamond a \wedge \diamond b \neq \perp$.

We can describe the set $\left\{x / R^{-1}(x) \neq \varnothing\right\}$ of Theorem 2.6 in a topological way as the closure in the Stone topology of the set of points with nonempty $\bar{T}$-derivate set (see proof of Theorem 2.3). In this way we obtain a purely topological characterization of the disjunction property, but still the preceding theorems are quite unnatural. In the next section we will concentrate in the direction of the class of algebras that most concern us, the algebras of theories: we will see that the use of the topology $\bar{T}$ becomes more natural, and allows us to obtain characterizations of relevant logical properties of theories (see Corollary 3.5. Theorem 4.3]. Already in the case of diagonalizable algebras without atoms, things become simpler.

We recall that a Boolean algebra is atomless iff there are no open points in the dual space, or equivalently, iff for each point of the dual space there exists a net $\left(y_{\alpha}\right)_{\alpha \in I}$ of elements different from $y$ that converges to $y$ in the Stone topology.

\section{$3 \quad \Sigma_{1}$-Sound theories and compactness}

Theorem 3.1 If a diagonalizable algebra D is atomless, the following conditions are equivalent:

(i) D has the strong disjunction property;

(ii) the closed sets in $\left(D^{*}, \bar{T}\right)$ are connected;

(iii) every net of $\left(D^{*}, \bar{T}\right)$ converges.

Proof: (i) $\rightarrow$ (ii) This follows from Theorem 2.8 .

(ii) $\rightarrow$ (iii) From (ii) it follows that there is at most one point $z$ with $R^{-1}(z)=\varnothing$. If there is such a $z$, and $x \neq z$, we must have $z R x$; thus, every point of the dual space belongs to the set $z \cup R(z)$ and every net converges to $z$. Now suppose that $R^{-1}(x) \neq$ $\varnothing$ for each $x \in D^{*}$; if $x, y \in D^{*}$, then $R^{-1}(x) \bigcap R^{-1}(y) \neq \varnothing$, otherwise the closed set $R^{-1}(x) \cup R^{-1}(y)$ would be disconnected. It follows that for every natural $n$ and $x_{1}, \ldots, x_{n} \in D^{*}$, the set $R^{-1}\left(x_{1}\right) \cap \ldots \cap R^{-1}\left(x_{n}\right)$ is not empty. The sets $R^{-1}\left(x_{i}\right)$ are closed in the Stone topology and the Stone space is compact, thus $\bigcap_{x \in D^{*}} R^{-1}(x) \neq$ $\varnothing$. If $z \in \bigcap_{x \in D^{*}} R^{-1}(x)$, every net in $\left(D^{*}, \bar{T}\right)$ converges to $z$.

(iii) $\rightarrow$ (i) We will first show that $R^{-1}(z) \neq \varnothing$ for each $z$ in the space, that is, the algebra $D$ is $\omega$-consistent. Assume $R^{-1}(z)=\varnothing$, and let $u$ be a point different from $z$ : the sequence that takes alternatively the values $u$ and $z$ must converge to $z$ and so $z R u$. Since the algebra is atomless, there is a net $\left(z_{\alpha}\right)$, consisting of elements different from $z$, that converges to $z$ in the Stone topology; but $z R u$ for each $u \neq z$, thus the net converges to $z$ also in the $\bar{T}$ topology, and $z R z$ according to Proposition 1.7. this contradicts $R^{-1}(z)=\varnothing$. The disjunction property of the algebra follows from Theorem 2.6 .

Remark 3.2 We do not use the hypothesis that the algebra is atomless to prove (i) $\rightarrow$ (ii) and (ii) $\rightarrow$ (iii); moreover without this hypothesis we can prove (iii) $\rightarrow$ (ii).

Indeed, let $C$ be a closed, nonempty disconnected set, and let $C_{1}$ and $C_{2}$ be two closed nonempty sets such that $C=C_{1} \cup C_{2}$ and $C_{1} \cap C_{2}=\varnothing$. If $x \in C_{1}$ and 
$y \in C_{2}$, let $\left(z_{n}\right)$ be the sequence that alternatively takes the values $x, y$. If this sequence converges to a point $z$, then the two constant subsequences with values $x$ and $y$ converge to $z$ too, and so $z \in C_{1} \cap C_{2}$, contradicting the fact that $C_{1} \cap C_{2}$ is empty. The hypothesis of being an atomless algebra, however, is necessary for (iii) $\rightarrow$ (i): we obtain a counterexample by considering a Boolean algebra containing an atom $a$, endowed with the $\square$ operator that is obtained in the usual way from the relation $R=\left\{(a, x) / x \neq a, x \in D^{*}\right\}$ defined in the dual space of the algebra (where we identify the atom $a$ with the principal ultrafilter generated by it).

The topology $\bar{T}$ defined from $R$ has the property that every net converges to the point $a$; nevertheless, from Lemma 2.4 we see that the diagonalizable algebra defined in this way has not the strong disjunction property.

From Theorem 3.1 and Remark 3.2 we see that the dual $\bar{T}$-space of a diagonalizable algebra with the strong disjunction property is certainly compact. Our next step will be to show that, under certain hypotheses, the compactness of the $\bar{T}$-dual space implies the strong disjunction property of the diagonalizable algebra. In general, this is not true, as Remark 3.2 hhows.

Moreover, the hypothesis of being atomless does not help in this case: we next describe an atomless ( $\omega$-consistent) diagonalizable algebra without the strong disjunction property that has a $(\bar{T})$-compact dual space. Consider the diagonalizable algebra of Peano Arithmetic, $D_{P A}$, and the direct product $D_{P A} \times D_{P A}$. The dual space is the disjoint union of two copies of the dual of $D_{P A}$ (see D'Agostino [4]), and it is $\bar{T}$-compact because $D_{P A}^{*}$ is so according to Theorem 3.1. while the algebra is $\omega$-consistent and atomless. Nevertheless, the algebra has not the (strong) disjunction property. This means that in order to prove that the compactness of the space $\left(D^{*}, \bar{T}\right)$ implies the strong disjunction property, we have to consider a smaller class of algebras than the atomless ones. However, we can prove:

Theorem 3.3 If $D$ is atomless and $\left(D^{*}, \bar{T}\right)$ is compact, then $D$ is $\omega$-consistent.

Proof: We shall prove, using Proposition 1.7, that for each $y$ in $D^{*}$ the set $R^{-1}(y)$ is not empty.

Since the algebra is without atoms, for each $y$ in the dual space there is a net $\left(y_{\alpha}\right)_{\alpha \in I}$ of elements different from $y$ that converges to $y$ in the Stone space. If $\left(D^{*}, \bar{T}\right)$ is compact, there is a subnet $\left(y_{\alpha}\right)_{\alpha \in J}$ of $\left(y_{\alpha}\right)_{\alpha \in I}$ that converges in $\left(D^{*}, \bar{T}\right)$ to some point $x$. If $y_{\alpha} \neq x$ for each $\alpha \in J$, we have $x R y$ (see Proposition 1.7). In any case, there must be a $\delta \in J$ such that for each $\gamma \in J, \gamma \succ \delta, y_{\gamma}$ is different from $x$; otherwise we could find a subnet of $\left(y_{\alpha}\right)_{\alpha \in J}$ with constant value $x$. Then, however, $\left(y_{\alpha}\right)_{\alpha \in J}$ would converge in the Stone topology to $x$ and $x$ would be equal to $y$, because the Stone topology is $T_{1}$. This is in contradiction with the choice of the net $\left(y_{\alpha}\right)_{\alpha \in I}$. In this way we can prove the existence of a net of elements, different from $x$, that converges to $x$ in the topology $\bar{T}$ and to $y$ in the Stone topology. We then conclude $R^{-1}(y) \neq \varnothing$ for each $y$ in the dual space, and the algebra is $\omega$-consistent.

Remark 3.4 If the algebra contains atoms, Theorem 3.3 s not true, even for infinite algebras. Indeed, consider the example in Remark 3.2 where the space $\left(D^{*}, \bar{T}\right)$ is compact while the algebra is not $\omega$-consistent.

Since the Lindenbaum algebra of an arithmetical theory is atomless, we obtain: 
Corollary 3.5 The following conditions are equivalent:

(i) the theory $S$ is $\Sigma_{1}$-sound;

(ii) all closed sets in $\left(D_{S}^{*}, \bar{T}\right)$ are connected;

(iii) every net in $\left(D_{S}^{*}, \bar{T}\right)$ is convergent;

(iv) the space $\left(D_{S}^{*}, \bar{T}\right)$ is compact.

Proof: (i) $\leftrightarrow$ (ii), (i) $\leftrightarrow$ (iii) follow from Theorem 3.1 and Proposition 2.2.

(iii) $\rightarrow$ (iv) is trivial.

(iv) $\rightarrow$ (i) follows by applying Theorem 3.3 . because in the case of Lindenbaum diagonalizable algebra the $\omega$-consistency of the algebra implies the $\Sigma_{1}$-soundness of the theory (see Proposition 2.2).

4 Admissible elements We now want to characterize sentences that are $\Sigma_{1}$-conservative over a $\Sigma_{1}$-sound theory $S$.

We recall that an element $a$ of a diagonalizable algebra $D$ is said to be admissible (see [9]) if $a \leq \square b$ implies $b=\top$, or equivalently, $a \wedge \diamond b \neq \perp$, for every $b \neq \perp$.

Proposition 4.1 (See Shavrukov [9]). Let $S$ be a $\Sigma_{1}$-sound theory. A sentence a is $\Sigma_{1}$-conservative over $S$ iff $a$ is admissible in $D_{S}$; then the theory $S \cup\{a\}$ is still $\Sigma_{1}$-sound iff a is admissible.

Lemma 4.2 An element a of a diagonalizable algebra $D$ is admissible iff for each point $x$ of the dual space we have $R^{-1}(x) \cap a \neq \varnothing$.

Proof: Let $x$ be a point in $D^{*}$; if $a$ is admissible, the set $\{a \wedge \diamond b / b \in x\}$ has the finite meet property in the sense of Boolean algebras; indeed, we have $a \wedge \diamond b_{1} \wedge \ldots \wedge$ $\diamond b_{n} \geq a \wedge \diamond\left(b_{1} \wedge \ldots \wedge b_{n}\right)$, thus if $b_{1}, \ldots, b_{n} \in x$ and $a \wedge \diamond b_{1} \wedge \ldots \wedge \diamond b_{n}=\perp$, then $a \wedge \diamond\left(b_{1} \wedge \ldots \wedge b_{n}\right)=\perp$ and the admissibility of $a$ contradicts the hypothesis $b_{1} \wedge$ $\ldots \wedge b_{n} \in x$. If $z$ is an ultrafilter containing $\{a \wedge \diamond b / b \in x\}$, then $z \in R^{-1}(x) \cap a \neq \varnothing$.

Vice versa, if $b$ is not $\perp$, let $x$ be an ultrafilter containing $b$. From $R^{-1}(x) \cap a \neq$ $\varnothing$ it follows $a \wedge \diamond b \neq \perp$.

Theorem 4.3 In a diagonalizable algebra with the strong disjunction property, the following conditions are equivalent:

(i) a is admissible;

(ii) every net of $\left(D^{*}, \bar{T}\right)$ converges to a point in a;

(iii) every nonempty closed set of $\left(D^{*}, \bar{T}\right)$ has a nonempty intersection with a.

In particular, a sentence a in the language of PA is $\Sigma_{1}$-conservative over PA iff, considering the diagonalizable algebra $D=D_{P A}$, (ii) (or equivalently (iii)) holds.

Proof: (i) $\rightarrow$ (ii) Since the diagonalizable algebra has the strong disjunction property, if $x_{1}, \ldots, x_{n}$ are points in the dual space, using Lemma 4.2 we can prove $a \cap$ $R^{-1}\left(x_{1}\right) \cap \ldots \cap R^{-1}\left(x_{n}\right) \neq \varnothing$; but the Stone space is compact, and we deduce the existence of a point $z$ such that $z \in a$ and $z R x$ for each point $x$ in the space. This implies that every net of $\left(D^{*}, \bar{T}\right)$ converges in $a$.

(ii) $\rightarrow$ (iii) Easy. 
(iii) $\rightarrow$ (i) From the strong disjunction property we deduce that if $b \neq \perp$, then $\diamond b \neq \perp$; but $\diamond b$ is a $\bar{T}$-closed set and (iii) implies $a \wedge \diamond b \neq \perp$.

The Stone space of a diagonalizable algebra is always a $T_{1}$ space, while the topology $\bar{T}$ is $T_{1}$ iff the algebra is trivial (i.e., if $\square a=\top$ for each $a$ in the algebra). It is still possible for an algebra to have closed points, and we will see that this property is linked with the admissible elements. However, we will show that the Lindenbaum diagonalizable algebra of a $\Sigma_{1}$-sound theory does not have any closed point.

Theorem 4.4 If D has the strong disjunction property, the following conditions are equivalent:

(i) a $\bar{T}$-closed point exists (and only one according to Theorem 2.8;

(ii) the admissible elements form an ultrafilter $x_{0}$ (the closed point in (i));

(iii) $\bigcap\left\{C /\right.$ Cis closed and not empty in $\left.\left(D^{*}, \bar{T}\right)\right\}$ is a singleton (the ultrafilter $x_{0}$ in (ii)).

Proof: (i) $\rightarrow$ (ii) If the diagonalizable algebra has the strong disjunction property, then:

(a) the element $T$ is admissible;

(b) if $a \leq b$ and $a$ is admissible then $b$ is admissible;

(c) if $a$ is admissible then for each $b$, either $a \wedge b$ or $a \wedge \neg b$ is admissible (see [9]).

To prove that the admissible elements form an ultrafilter we have just to show that there is no $a$ such that both $a$ and $\neg a$ are admissible. If this were the case, using Lemma 4.2 we would have $R^{-1}\left(x_{0}\right) \cap \neg a \neq \varnothing$, and $R^{-1}\left(x_{0}\right) \cap a \neq \varnothing$, which is impossible if $x_{0}$ is closed.

(ii) $\rightarrow$ (iii) If $z \in \bigcap\left\{C / C\right.$ is closed and not empty in $\left.\left(D^{*}, \bar{T}\right)\right\}$, then $\diamond b \in z$ if $b \neq \perp$. If $a$ is admissible, $z$ does not contain $\neg a$, otherwise $\neg a \wedge \diamond b$ would be different from $\perp$ for each $b \neq \perp$ and $a, \neg a$ would both be admissible, contradicting the hypothesis that the admissible elements form an ultrafilter $x_{0}$. This implies that every admissible element is in $z$ and thus $z=x_{0}$. On the other hand, if $C$ is closed and not empty in $\left(D^{*}, \bar{T}\right)$, then, for each $x$ in $C, x_{0} \in R^{-1}(x)$, since the closed sets in $\left(D^{*}, \bar{T}\right)$ are connected (see Theorem 2.8).

(iii) $\rightarrow$ (i) The point $x_{0}$ is closed.

For example, the free algebra on the empty set has a unique closed point, the ultrafilter of cofinite sets. The situation is different in Lindenbaum algebras; indeed, since the set $\bigcap\left\{C / C\right.$ is closed and not empty in $\left.\left(D^{*}, \bar{T}\right)\right\}$ is the set of all ultrafilters that contain $\{\diamond b / b \neq \perp\}$, it has cardinality $2^{\aleph_{0}}$ (see Montagna [8) and from the preceding theorem it follows that the dual space does not have any closed point.

Admissible elements allow us to obtain a topological property that is shared by Lindenbaum diagonalizable algebras but not by the whole class of diagonalizable algebras. Let us consider finitely generated quotients of a Lindenbaum diagonalizable algebra $D_{S}$; these quotients correspond to diagonalizable algebras of theories $S+\varphi$, for a sentence $\varphi$ such that $S \vdash \varphi \rightarrow \operatorname{Teor}_{S}(\varphi)$ : in fact, we can choose the provability predicate of $S+\varphi$ to be $\operatorname{Teor}_{S}(\varphi \rightarrow x)$.

These quotients, when proper, cannot be $\omega$-consistent; indeed: 
$D_{S+\varphi}$ is $\omega$-consistent $\Rightarrow S+\varphi$ is $\Sigma_{1}$-sound $\Rightarrow \varphi$ is an admissible element of $D_{S}$ (according to Proposition 2.2 and Lemma4.1); but then from $\varphi \leq \square \varphi$ in $D_{S}$ we infer $\varphi=1$.

Note:

(a) finitely generated quotients correspond in the dual space to Stone's clopen that are open in $\bar{T}$ (see Bernardi and D'Aquino [2]);

(b) in Lindenbaum algebras these quotients are $\omega$-consistent iff the corresponding sets in the dual space are $\bar{T}$-compact (see Corollary 3.5 and Proposition 2.2.

From this we obtain :

Lemma 4.5 If $S$ is $\Sigma_{1}$-sound, in the dual space of the Lindenbaum algebra $D_{S}$ there are no nontrivial clopen sets that are both open and compact in the $\bar{T}$-topology.

By considering the dual space of the free algebra on the empty set, we see that this result is not true in the class of all diagonalizable algebras.

\section{REFERENCES}

[1] Abashidze, M., and L. Esakia, "Cantor's scattered spaces and logic of provability," Baku International Topological Conference, Abstracts of Reports, Part 1 (1987), p. 3.

[2] Bernardi, C., and P. D'Aquino, "Topological duality for diagonalizable algebras," Notre Dame Journal of Formal Logic, vol. 29 (1988), pp. 345-364.Zbl 0655.03041 MR 89i:03114 1.4.

[3] Blass, A., "Infinitary combinatorics and modal logic," Journal of Symbolic Logic, vol. 55 (1990), pp. 771-778. Zb1 0699.03008MR 91k:03123 1

[4] D'Agostino, G., "Reflexive points in the topological study of diagonalizable algebra," Bollettino Unione Matematica Italiana, vol. 6-B (1992), pp. 689-701.Zbl 0773.03040 MR 94h:03129 1,11,3

[5] Friedman, H., "The disjunction property implies the numerical existence property," Proceedings of the National Acadamy of Sciences U.S.A., (1975), pp. 2877-2878. Zbl 0342.02012||MR 52:47

[6] Magari, R., "The diagonalizable algebras," Bollettino Unione Matematica Italiana, vol. 12 (1975), pp. 117-125. Zbl 0352.08009|MR 57:105 1

[7] Magari, R., "Representation and duality theory for diagonalizable algebras," Studia Logica, vol. 34 (1975), pp. 305-313. Zbl 0355.02021|MR 57:107 1

[8] Montagna, F., "On the Diagonalizable Algebra of Peano Arithmetic," Bollettino Unione Matematica Italiana vol. 16-B (1979), pp. 795-813.ZZbl 0419.08010||MR 81d:03065]4

[9] Shavrukov, V., "Subalgebras of diagonalizable algebras of theories containing arithmetic," Dissertationes Mathematicae, vol. 323 (1993). Zbl 0803.03044|MR 94h:03131 2.12.2.2.4.4.1.4.

[10] Shavrukov, V., "A note on the diagonalizable algebras of $P A$ and $Z F$," Annals of Pure and Applied Logic, vol. 61 (1993), pp. 161-173.Zbl 0780.03027|MR 94h:03132 1

Università degli Studi di Udine

Dipartimento di Matematica e Informatica 
via Zanon 6

33100 Udine

Italy

email:dagostin@dimi.uniud.it 